\title{
On the Performance of Sliding Window TD-LMMSE Channel Estimation for 5G Waveforms in High Mobility Scenario
}

\author{
Michel Saideh $^{1}$, Student Member, IEEE, Marion Berbineau ${ }^{1}$, Member, IEEE, Iyad Dayoub ${ }^{2}$, Senior \\ Member, IEEE \\ ${ }^{1}$ Univ. Lille Nord de France, IFSTTAR, COSYS, F-59650 Villeneuve d'Ascq \\ ${ }^{2}$ Univ. Valenciennes, CNRS, Univ. Lille, YNCREA, Centrale Lille, UMR 8520 - IEMN, DOAE, F-59313 \\ Valenciennes, France
}

\begin{abstract}
The upcoming $5 \mathrm{G}$ era is considered as a critical answer to future railway communication needs. In this work, we analyze the performance of sliding window time domain LMMSE channel estimation technique where a generalized development is proposed and analyzed for 5G FBMC-(O)QAM waveform candidates. In order to enhance performance, the analytical model is extended to exploit multipaths and multiantennas correlation wherever they do exist e.g., tunnels. TD-LMMSE performance for 5G waveforms and achieved enhancements are verified by Monte Carlo simulations.
\end{abstract}

Index Terms - 5G, FBMC, LMMSE, Channel Estimation

\section{INTRODUCTION}

For the future railway communications related to control and command, the upcoming 5G system where low latency, high mobility, efficient spectrum reuse and asynchronous communications should be supported, is considered as a possible answer for the replacement of the GSM-R system. However, choosing a suitable $5 \mathrm{G}$ waveform isn't a straightforward task which has led to the proposal of many $5 \mathrm{G}$ waveform candidates [1]-[5]. The filtering operation has been widely adopted to avoid the bad frequency localization of the 4G Orthogonal Frequency Division Multiplexing (OFDM) waveform. Hence, a group of candidates have adopted the idea of filtering each subcarrier on its own e.g. Filter Bank Multi Carrier (FBMC) [1], [2], thus offering outstanding spectral containment enhancements. While others have proposed to apply filtering on each group of subcarriers, e.g. Universal Filtered (UF)-OFDM [4], hence offering tradeoffs between time and frequency localizations. Direct modifications have been also applied upon 4G DFTspread-OFDM waveforms, thus guarding a high backward compatibility e.g. Generalized DFT-spread-OFDM [5].

The highly contained spectrum of FBMC has made it highly suited for efficient spectrum reuse and asynchronous communications. However, the FBMC-QAM version has a loss in its spectral efficency while guards complex orthogonality between its subcarriers. On the other hand, the FBMC spectrally efficient version, the FBMC-Offset QAM (OQAM), suffers from a loss of orthogonality in the complex domain which introduces a new kind of interference, the so called intrinsic interference, that requires special treatment to deal with it. Hence, many interference cancellation schemes at pilot positions have been proposed such as using auxilary pilots [6] or some precoding techniques [7].

In high speed scenarios, channel estimation becomes more challenging due to the Doppler induced Inter Carrier Interference (ICI). While preamble based channel estimation [8] is not suited for high speed scenarios, some scattered pilot patterns have been adopted recently for FBMC in Pilot Aided Channel Estimation (PACE) context. [9] applied a least square estimation at pilot positions while Minimum Mean Square Error (MMSE) interpolation was used to estimate the channel at data positions. A channel adaptive modulation technique was adopted in [10] with linear interpolation.

In a previous work [11], we have proposed a Time DomainLMMSE (TD-LMMSE) channel estimation technique for OFDM that adopts a comb pilot pattern and estimates the channel impulse response at the middle of a sliding window. This combination has been able to exploit channel correlation of adjacent time samples within a window for the benefits of the sample in the middle, thus having expelling performance in high speed scenarios. In this paper, we generalize the sliding window TD-LMMSE technique and analyze it for $5 \mathrm{G}$ FBMC-(O)QAM waveforms. Analytical development is also proposed to exploit the multipaths and multiantennas correlation, whenever they do exist e.g. tunnels [12], High Speed Railways (HSR) [13] and Vehicule to Vehicule (V2V) [14] environments, thus enhancing performance. Monte Carlo simulations are conducted to verify the analytical development.

This paper is organized as follows. Section II describes the FBMC technique while section III proposes the analytical analysis of TD-LMMSE. Section IV includes the simulation part. The work is concluded in section V.

\section{A. Notations}

In the following, bold lowercase variables $c$ represent vectors, bold uppercase variables $C$ represent matrices. $C^{H}$ is the Hermitian conjugate of $\boldsymbol{C}$. We use Matlab notations where for example $C(1,:)$ means the first row of $C$. The function $\operatorname{diag}(\boldsymbol{x})$ creates a diagonal matrix with the vector $\boldsymbol{x}$ as its diagonal. $\odot$ is the element-wise product. 


\section{FilterBANK MULTiCARRIER WAVEFORM}

The time domain transmitted signal of a multicarrier system could be written as:

$$
x(t)=\sum_{k=0}^{K-1} \sum_{z=0}^{Z-1} g_{z, k}(t) s_{z, k}
$$

where $s_{z, k}$ is the symbol transmitted at the $z$ subcarrier and the $k$ time position.

$$
g_{z, k}(t)=f(t-k T) e^{j 2 \pi z F(t-k T)}
$$

$T$ and $F$ are the time and frequency spacings. $g_{z, k}(t)$ is the prototype filter $f(t)$ shifted by $k T$ in time and by $z F$ in frequency.

In discrete time domain, a matrix form of equation (1) could be written as:

$$
\boldsymbol{x}=\boldsymbol{G s}
$$

Where

$$
\begin{gathered}
\boldsymbol{G}=\left[\boldsymbol{g}_{1,1} \boldsymbol{g}_{2,1} \ldots \boldsymbol{g}_{Z, 1} \boldsymbol{g}_{1,2} \ldots \boldsymbol{g}_{Z, K}\right] \\
\boldsymbol{s}=\left[s_{1,1} s_{2,1} \ldots s_{Z, 1} s_{1,2} \ldots s_{Z, K}\right]^{T}
\end{gathered}
$$

$\boldsymbol{g}_{z, k}$ is the $N \times 1$ vector formulated by sampling $g_{z, k}(t)$ in equation (2) and gathering the samples within the vector $\boldsymbol{g}_{z, k}$. $N$ is the number of samples of the whole transmission block.

The fact that OFDM sacrifies the Time Frequency Localization (TFL) property of the Balian Low Theorem (BLT) [15] theorem to achieve the complex orthogonality $\boldsymbol{G}^{H} \boldsymbol{G}=\boldsymbol{I}$ and the full spectral efficiency $T F=T_{0} F_{0}=1$ conditions, where $T_{0}=1 / F_{0}$ represents the maximal case, has caused major problems in asychronous communications and efficient spectrum reuse applications. Filter bank based waveforms on the other hand are concerned with achieving the TFL advantage by sacrificing other properties. Spectral density of $T F=1$ could be sacrificed for example hence permitting us of adopting a well TFL prototype filter within the MC technique. While different FBMC-QAM versions have been proposed [1], [2], the one that sacrifices the spectral density by increasing the spacing between adjacent subcarriers " $F=2 / T$ " [1] is adopted in our study since it guards the high frequency localization property and exhibits less ICI which is important in high mobility scenarios and in the study of the time domain LMMSE technique. Different TFL prototype filters could be used in that regard e.g. Hermite, PHYDYAS [16], Root Raised Cosine (RRC) where each of which has different time frequency localization properties.

A more known FBMC version is the FBMC-OQAM one [1], which guards the full spectral density feature $T F=1$ and the TFL property by sacrificing the orthogonality condition. FBMC-OQAM could be considered as a modified version of the FBMC-QAM where it has the same prototype filter that is designed to be orthogonal for $T=T_{0}$ and $F=2 / T_{0}$. However, the following modifications are made:

- Only real symbols are transmitted "PAM"

- Time frequency spacing is reduced to $T=T_{0} / 2$ and $F=1 / T_{0}$.
- A new phase shift component " $e^{j \theta_{z, k}}$ " is added to equation (2) where $\theta_{z, k}=\frac{\pi}{2}(z+k)$. This is used to shift the interference components, that are added due to the loss of orthogonality, into the imaginary domain. Hence, a new orthogonality condition which stands in the real domain is introduced: $\Re\left\{\boldsymbol{G}^{H} \boldsymbol{G}\right\}=\boldsymbol{I}$ where $\boldsymbol{R}\{x\}$ is the real part of $x$.

\section{GENERALIZED SLIDING WINDOW TD-LMMSE}

The sliding window time domain LMMSE channel estimator is based upon estimating the channel impulse response at the receiver by gathering a window of $N_{w}$ successive received samples in the time domain and estimating the channel impulse response at the middle of this window by using the LMMSE technique. That allows to exploit the correlation between received samples, at both sides of the desired sample, to get a better channel estimate, as it is explained in details in [11]. Then, we slide the window one sample, generate the new LMMSE filter, estimate the mid sample, and so on.

In high mobility scenarios, adopting the comb type pilot pattern has shown in our previous work to be preferred than the block type [11], hence it is adopted in our study also.

By writing the data and pilot symbols independently, equation (1) could be rewritten in a discrete form as:

$$
x(n)=\sum_{k=0}^{K-1} \sum_{z=0}^{Z-1} g_{z, k}(n)\left(p_{z, k}+d_{z, k}\right)
$$

Where $d_{z, k}$ and $p_{z, k}$ are the data and pilot symbols, respectively, transmitted at the $z$ subcarrier and the $k$ time position. $d_{z, k}\left(p_{z, k}\right)$ is equal to zero when $\{z, k\}$ refers to a slot used for pilot (data) symbols. Eq. (6) could be rewritten as:

$$
x(n)=x_{p}(n)+x_{d}(n)
$$

where

$$
\begin{aligned}
& x_{p}(n)=\sum_{k=0}^{K-1} \sum_{z=0}^{Z-1} g_{z, k}(n) p_{z, k} \\
& x_{d}(n)=\sum_{k=0}^{K-1} \sum_{z=0}^{Z-1} g_{z, k}(n) d_{z, k}
\end{aligned}
$$

$x_{p}(n)$ is the $n$th sample of the time domain pilot signal which is used to estimate the channel. $x_{d}(n)$ is the $n$th sample of the time domain data signal that acts as an interference in the estimation process.

Passing through a time variant multipath channel, the $n$th sample of the received signal could be written as:

$$
y(n)=\sum_{l=0}^{L-1} h(n, l) x(n-l)+w(n)
$$

Where $h(n, l)$ is the $n t h$ time domain channel sample of the $l$ th path. $L$ is the total number of paths which is assumed to be the same over the time domain. $w(n)$ is the additive white Gaussian noise.

In order to estimate the channel sample $h(k, l)$, we have first to collect the $N_{w}$ surrounding samples of the $k$ th received sample i.e. $\boldsymbol{y}_{k}=\left[y\left(k-N_{w} / 2\right) \ldots y\left(k+N_{w} / 2-1\right)\right]^{T}$. In what 
follows we omit the subscript $k$ for simplicity. Using (10) we can write $\boldsymbol{y}$ as:

$$
\boldsymbol{y}=\boldsymbol{A}\left(\sum_{l=0}^{L-1}\left(\boldsymbol{X}_{p_{l}}+\boldsymbol{X}_{d_{l}}\right) \boldsymbol{h}_{l}+\boldsymbol{w}\right)
$$

$\boldsymbol{A}$ is a mapping matrix used in the CP-OFDM waveform to remove the cyclic prefix. $\boldsymbol{X}_{p_{l}}=\operatorname{diag}\left(\boldsymbol{x}_{p_{l}}\right)$ where $\boldsymbol{x}_{p_{l}}=$ $\left[x_{p}\left(k-N_{w} / 2-l\right) \ldots x_{p}\left(k+N_{w} / 2-1-l\right)\right]^{T}$. The same definition is used for $\boldsymbol{X}_{d_{l}}$ and $\boldsymbol{x}_{d_{l}} . \boldsymbol{h}_{l}=\left[h\left(k-N_{w} / 2, l\right) \ldots h\left(k+N_{w} / 2-\right.\right.$ $1, l)]^{T}, \boldsymbol{w}=\left[w\left(k-N_{w} / 2\right) \ldots w\left(k+N_{w} / 2-1\right)\right]^{T}$.

$$
\begin{aligned}
& \boldsymbol{x}_{d_{l}}=\boldsymbol{G}_{l} \boldsymbol{d} \\
& \boldsymbol{x}_{p_{l}}=\boldsymbol{G}_{l} \boldsymbol{p}
\end{aligned}
$$

Where $\boldsymbol{d}=\left[d_{1,1} d_{2,1} \ldots d_{Z, 1} d_{1,2} \ldots d_{Z, K}\right]^{T}, \quad \boldsymbol{p}=$ $\left[p_{1,1} p_{2,1} \ldots p_{Z, 1} p_{1,2} \ldots p_{Z, K}\right]^{T}$, and $\boldsymbol{G}_{l}=\boldsymbol{G}\left(k-N_{w} / 2-l:\right.$ $\left.k+N_{w} / 2-1-l,:\right)$.

Using (11) the TD-LMMSE estimates the channel sample $h(k, l)$ using the following equation:

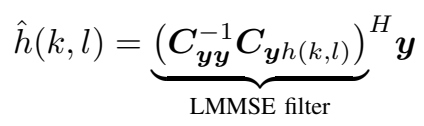

Where $\boldsymbol{C}_{\boldsymbol{y} \boldsymbol{y}}=\boldsymbol{E}\left[\boldsymbol{y} \boldsymbol{y}^{H}\right]$ is the covariance matrix of the received window $\boldsymbol{y} . \boldsymbol{C}_{\boldsymbol{y} h(k, l)}=\boldsymbol{E}[\boldsymbol{y} h(k, l)]$ is the correlation matrix between the received window $\boldsymbol{y}$ and the channel sample $h(k, l)$.

Assuming a Wide Sense Stationary Uncorrelated Scattering (WSSUS) channel we can write:

$$
\begin{gathered}
\boldsymbol{C}_{\boldsymbol{y} \boldsymbol{y}}=\boldsymbol{A}\left(\sum_{l=0}^{L-1}\left[\boldsymbol{R} p_{l, l}+\boldsymbol{R} d_{l, l}\right] \odot \boldsymbol{R}_{\boldsymbol{h}_{l} \boldsymbol{h}_{l}}+\boldsymbol{R}_{w}\right) \boldsymbol{A}^{H} \\
\boldsymbol{C}_{\boldsymbol{y} h(k, l)}=\boldsymbol{A} \boldsymbol{X}_{p_{l}} \boldsymbol{R}_{\boldsymbol{h}_{l} \boldsymbol{h}_{l}}(:, k)
\end{gathered}
$$

Where eq. (15) has been written by noting that

$$
\operatorname{diag}(\boldsymbol{a}) \boldsymbol{B} \operatorname{diag}\left(\boldsymbol{a}^{H}\right)=\boldsymbol{a} \boldsymbol{a}^{H} \odot \boldsymbol{B}
$$

$\boldsymbol{R} p_{l_{1}, l_{2}}=\boldsymbol{x}_{p_{l_{1}}} \boldsymbol{x}_{p_{l_{2}}}^{H}, \boldsymbol{R}_{w}=E\left[\boldsymbol{w} \boldsymbol{w}^{H}\right]$ which is a diagonal matrix with noise variance in its diagonal vector. The $N_{w} \times N_{w}$ channel covariance matrix $\boldsymbol{R}_{\boldsymbol{h}_{l} \boldsymbol{h}_{l}}=E\left[\boldsymbol{h}_{l} \boldsymbol{h}_{l}^{H}\right]$ is a Toepltiz matrix with its first row elements given from Jakes' model for different time samples $\left(n_{1}, n_{2}\right)$ :

$$
\boldsymbol{R}_{\boldsymbol{h}_{l} \boldsymbol{h}_{l}}\left(1,\left|n_{2}-n_{1}\right|\right)=\alpha_{l} J_{0}\left(2 \pi\left(\left|n_{2}-n_{1}\right|\right) T_{s} f_{m}\right)
$$

Where $\alpha_{l}$ depends upon the channel power delay profile. $J_{0}()$ is the Bessel function of the first kind with zero order, $T_{s}$ is the sampling period and $f_{m}$ is the maximum Doppler spread. $\boldsymbol{R} d_{l_{1}, l_{2}}=E\left[\boldsymbol{x}_{d_{l_{1}}} \boldsymbol{x}_{d_{l_{2}}}^{H}\right]=\boldsymbol{G}_{l_{1}} \boldsymbol{D} \boldsymbol{G}_{l_{2}}^{H}$, where $D=E\left[\boldsymbol{d} \boldsymbol{d}^{H}\right]$ is a diagonal matrix with its diagonal elements being equal to the variance of the data symbols at data positions while zeros at pilot positions.

\section{A. Multipaths correlation}

The assumption of uncorrelated multipaths is not suitable for all scenarios. Recent studies have shown a good amount of correlation among multipaths in mobile scenarios such as the HSR [13] and the (V2V) [14] environments. Hence, TDLMMSE performance could be enhanced by exploiting the channel correlated samples within different multipaths.

By adopting a correlated multipaths scenario, equations (15) and (16) could be reformulated as follows:

$$
\boldsymbol{C}_{\boldsymbol{y} \boldsymbol{y}}=\boldsymbol{A}\left(\sum_{l_{1}=0}^{L-1} \sum_{l_{2}=0}^{L-1}\left[\boldsymbol{R} p_{l_{1}, l_{2}}+\boldsymbol{R} d_{l_{1}, l_{2}}\right] \odot \boldsymbol{R}_{\boldsymbol{h}_{l_{1}} \boldsymbol{h}_{l_{2}}}+\boldsymbol{R}_{w}\right) \boldsymbol{A}^{H}
$$

$$
\boldsymbol{C}_{\boldsymbol{y} h\left(k, l_{1}\right)}=\boldsymbol{A} \sum_{l_{2}=0}^{L-1} \boldsymbol{X}_{p_{j}} \boldsymbol{R}_{\boldsymbol{h}_{l_{1}} \boldsymbol{h}_{l_{2}}}(:, k)
$$

For simplicity, we guard Jake's formula of eq. (18) while adding multipaths correlation as follows:

$\boldsymbol{R}_{\boldsymbol{h}_{l_{1}} \boldsymbol{h}_{l_{2}}}\left(1,\left|n_{2}-n_{1}\right|\right)=\rho_{l_{1}, l_{2}} \alpha_{l_{1}} \alpha_{l_{2}} J_{0}\left(2 \pi\left(\left|n_{2}-n_{1}\right|\right) T_{s} f_{m}\right)$

where $\rho_{l_{1}, l_{2}}$ is the multipaths correlation coefficient between the paths $l_{1}$ and $l_{2}$. The channel estimate $\hat{h}(k, l)$ is given by eq. (14).

\section{B. Multi Antenna Correlation}

In a similar way, by assuming a SIMO (Single Input Multiple Output) system with $M$ antennas at the receiver, the correlation between the received signals of different antennas, which exists in different scenarios such as in tunnels [12], could be exploited thus enhancing the TD-LMMSE performance. The overall received window could be written as:

$$
\tilde{\boldsymbol{y}}=\tilde{\boldsymbol{A}}\left(\sum_{l=0}^{L-1}\left(\tilde{\boldsymbol{X}}_{p_{l}}+\tilde{\boldsymbol{X}}_{d_{l}}\right) \tilde{\boldsymbol{h}}_{l}+\tilde{\boldsymbol{w}}\right)
$$

where $\tilde{\boldsymbol{y}}=\left[\boldsymbol{y}_{1}^{T} \boldsymbol{y}_{2}^{T} \ldots \boldsymbol{y}_{M}^{T}\right]^{T}$ and $\boldsymbol{y}_{m}$ is the received window $\boldsymbol{y}$ from the $m$ th antenna. $\tilde{\boldsymbol{X}}_{x_{l}}=\operatorname{diag}\left(\tilde{\boldsymbol{x}}_{x_{l}}\right)$ where $\tilde{\boldsymbol{x}}_{x_{l}}=$ $[\underbrace{\boldsymbol{x}_{x_{l}}^{T}}_{1} \underbrace{\boldsymbol{x}_{x_{l}}^{T}}_{2} \ldots \underbrace{\boldsymbol{x}_{x_{l}}^{T}}_{\mathrm{M}}]^{T} . \tilde{\boldsymbol{h}}_{l}=\left[\boldsymbol{h}_{l, 1}^{T} \boldsymbol{h}_{l, 2}^{T} \ldots \boldsymbol{h}_{l, M}^{T}\right]^{T}$ where $\boldsymbol{h}_{l, m}$ is the channel vector $\boldsymbol{h}_{l}$ of the $m$ th antenna.

Hence, the covariance matrices of the overall received window in (22) could be written as:

$$
\boldsymbol{C}_{\tilde{\boldsymbol{y}} \tilde{\boldsymbol{y}}}=\tilde{\boldsymbol{A}}\left(\sum_{l_{1}=0}^{L-1} \sum_{l_{2}=0}^{L-1}\left[\boldsymbol{R} \tilde{p}_{l_{1}, l_{2}}+\boldsymbol{R} \tilde{d}_{l_{1}, l_{2}}\right] \odot \boldsymbol{R}_{\tilde{\boldsymbol{h}}_{l_{1}} \tilde{\boldsymbol{h}}_{l_{2}}}+\boldsymbol{R}_{\tilde{\boldsymbol{w}}}\right) \tilde{\boldsymbol{A}}^{H}
$$

$$
\boldsymbol{C}_{\tilde{\boldsymbol{y}} h\left(k, l_{2}, m\right)}=\tilde{\boldsymbol{A}}\left(\sum_{l_{1}=0}^{L-1} \tilde{\boldsymbol{X}}_{p_{l_{1}}} \boldsymbol{R}_{\tilde{\boldsymbol{h}}_{l_{1}} \tilde{\boldsymbol{h}}_{l_{2}}}(:, k m)\right)
$$

Where $h(k, l, m)$ is the $k$ th channel sample of the $l$ th path and the $m$ th antenna. $\boldsymbol{R} \tilde{p}_{l_{1}, l_{2}}=\tilde{\boldsymbol{x}}_{p_{l_{1}}} \tilde{\boldsymbol{x}}_{p_{l_{2}}}^{H}, \boldsymbol{R} \tilde{d}_{l_{1}, l_{2}}=E\left[\tilde{\boldsymbol{x}}_{d_{l_{1}}} \tilde{\boldsymbol{x}}_{d_{l_{2}}}^{H}\right]$,

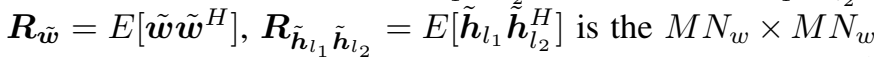
channel covariance matrix which is a combination of $M^{2}$ 
Toeplitz matrix where the first row elements of each are given for different $n_{2}, n_{1}$ values by:

$$
\boldsymbol{R}_{\tilde{\boldsymbol{h}}_{l_{1}} \tilde{\boldsymbol{h}}_{l_{2}}}\left(m_{1}, m_{2}\left|n_{2}-n_{1}\right|\right)=\beta_{l_{1} l_{2} m_{1} m_{2}} J_{0}\left(2 \pi\left(\left|n_{2}-n_{1}\right|\right) T_{s} f_{m}\right)
$$

Where

$$
\beta_{l_{1} l_{2} m_{1} m_{2}}=\gamma_{m_{1}, m_{2}} \rho_{l_{1}, l_{2}} \alpha_{l_{1}} \alpha_{l_{2}}
$$

$\gamma_{m_{1}, m_{2}}$ is the correlation coefficient between the antennas $m_{1}$ and $m_{2}$. The estimated channel sample could be written as:

$$
\hat{h}(k, l, m)=\left(\boldsymbol{C}_{\tilde{\boldsymbol{y}} \tilde{\boldsymbol{y}}}^{-1} \boldsymbol{C}_{\tilde{\boldsymbol{y}} h(k, l, m)}\right)^{H} \tilde{\boldsymbol{y}}
$$

\section{Complexity analysis}

From equation (27) we can see that the calculation of $h(k, l, m)$ comes with a complexity of $\left(\left(M N_{w}\right)^{2}+L M N_{w}\right)$ where $\left(M N_{w}\right)^{2}$ corresponds to the calculation of $\boldsymbol{C}_{\tilde{\boldsymbol{y}} \tilde{\boldsymbol{y}}}^{-1} \tilde{\boldsymbol{y}}$ while $L M N_{w}$ corresponds to $\boldsymbol{C}_{\tilde{\boldsymbol{y}} h(k, l, m)}^{H}\left(\boldsymbol{C}_{\tilde{\boldsymbol{y}} \tilde{\boldsymbol{y}}}^{-1} \tilde{\boldsymbol{y}}\right)$. In the special cases of a SISO system and/or $\rho=0$, complexity could be calculated by substituting with $M=1$ and/or $L=1$, respectively.

To reduce complexity, which is mainly due to the matrix inversion, we have suggested in [11] to fix our window during each MC symbol. That means we calculate $C_{\tilde{\boldsymbol{y}} \tilde{\boldsymbol{y}}}^{-1}$ once for each symbol while $\boldsymbol{C}_{\tilde{\boldsymbol{y}} h(k, l, m)}$ is calculated on a sample basis. In addition, the use of a periodic pilot sequence means that $\boldsymbol{R} \tilde{p}_{l_{1}, l_{2}}$ is the same among symbols. Adding this to the fact that in some communication environments we have a fixed trajectory, e.g. trains, means that the channel statistics and SNR values would be similar each time the train passes by the same location. Hence, $\boldsymbol{C}_{\tilde{\boldsymbol{y}} \tilde{\boldsymbol{y}}}^{-1}$ could be calculated offline for different channel statistics and SNR values and stored in a database.

\section{Normalized mean square error}

The normalized mean square error could be written as [17]

$$
N M S E_{k, l, m}=\frac{\sigma_{h(k, l, m)}^{2}-\sigma_{\hat{h}(k, l, m)}^{2}}{\sigma_{h(k, l, m)}^{2}}
$$

where $\sigma_{h(k, l, m)}^{2}$ is the variance of $h(k, l, m)$ and

$$
\sigma_{\hat{h}(k, l, m)}^{2}=\boldsymbol{C}_{\tilde{\boldsymbol{y}}_{k} h(k, l, m)}^{H} \boldsymbol{C}_{\tilde{\boldsymbol{y}}_{k} \tilde{\boldsymbol{y}}_{k}}^{-1} \boldsymbol{C}_{\tilde{\boldsymbol{y}}_{k} h(k, l, m)}
$$

The average NMSE over one MC symbol is:

$$
N M S E=\frac{1}{N L M} \sum_{k, l, m} N M S E_{k, l, m}
$$

\section{Simulation}

In this section, we demonstrate the simulation part where we report the numerical results. We adopt Jakes's model to demonstrate the time varying nature of the channel with a normalized square power delay profile where $L=7$. We adopt a subcarrier spacing of $15 \mathrm{KHz}$, carrier frequency $f_{c}=2.5$ $\mathrm{GHz}$, pilot spacing of $S_{p}=6$, a window size of 3 OFDM symbols, a mobile speed of $400 \mathrm{~km} / \mathrm{h}$, and a $(1 \times 4)$ SIMO system are adopted. $Z=256$ and $K$ is chosen in a way to include all significant interferers. FBMC prototype filters are designed with an overlapping factor value of 4 . A cyclic prefix of six samples length and 3000 repetitions for Montecarlo simulations are used.

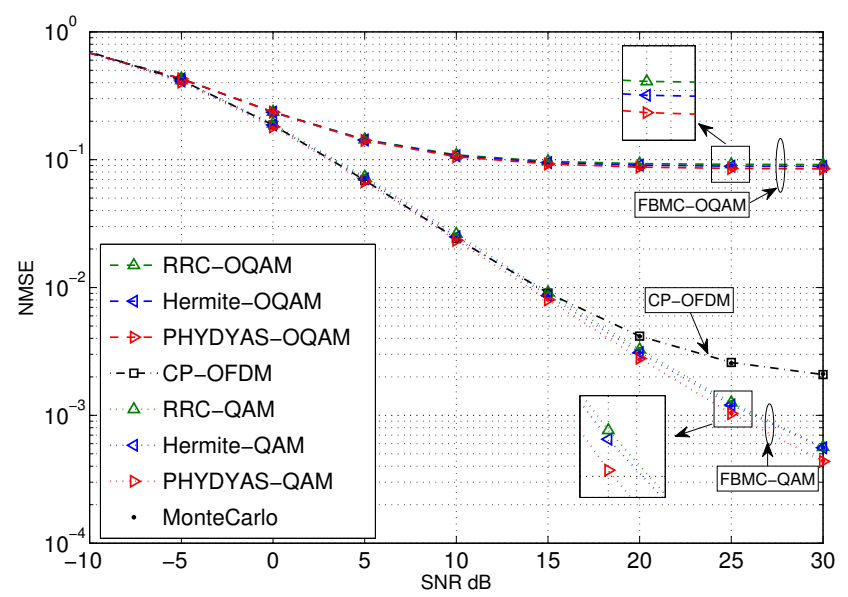

Figure 1. NMSE vs SNR for CP-OFDM and FBMC-(O)QAM with different prototype filters. WSSUS assumption and uncorrelated SIMO are adopted $(\rho=0, \gamma=0)$

In fig. 1 we show the theoretical and numerical performance of the TD-LMMSE technique for CP-OFDM and FBMC-(O)QAM where different prototype filters are adopted "PHYDYAS [16], Hermite, Root Raised Cosine (RRC)" with uncorrelated antennas and multipaths.

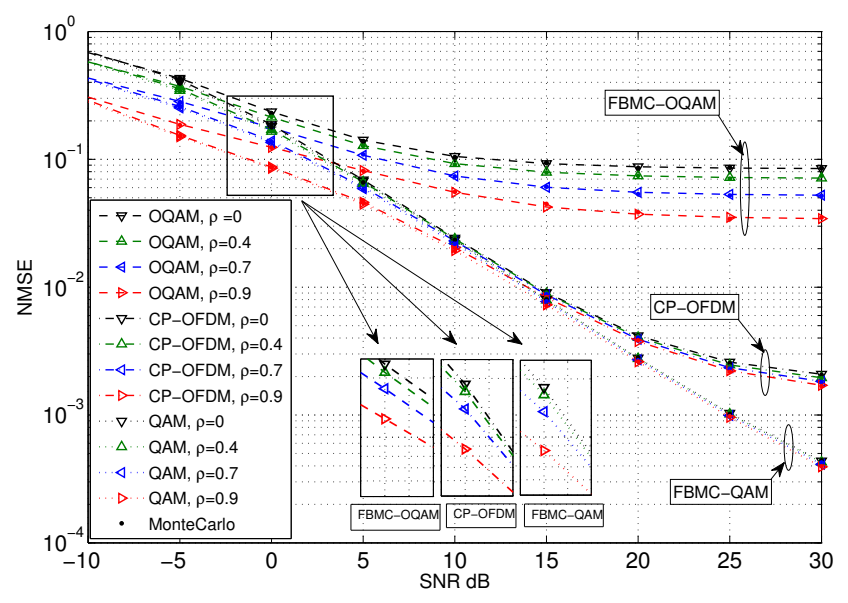

Figure 2. CP-OFDM and FBMC-(O)QAM performance with PHYDYAS and $\rho=0.4,0.7,0.9, \gamma=0$

We note that PHYDYAS prototype filter offers better performance than Hermite and RRC due to the fact that it is more localized in the frequency domain. Hence, we adopt the PHYDYAS filter in what follows. Fig.2 shows the enhancements in performance by the adoption of the multipaths correlation assumption. We assumed for simplicity that multipaths are correlated with each others by the same correlation coefficient $\rho$ and pictured the performance for different values of $\rho=0.4,0.7,0.9$. reasonable In fig. 3 we simulate the multi antennas correlation case. For clarity, we depict the performance of the FBMC-QAM only. We assume 
once again that antennas are correlated with each others with the same value $\gamma$ and we simulate the performance for different correlation values of $(\rho=0, \gamma=0.4,0.7,0.9)$. We note that the enhancement in performance fall within the low SNR region only.

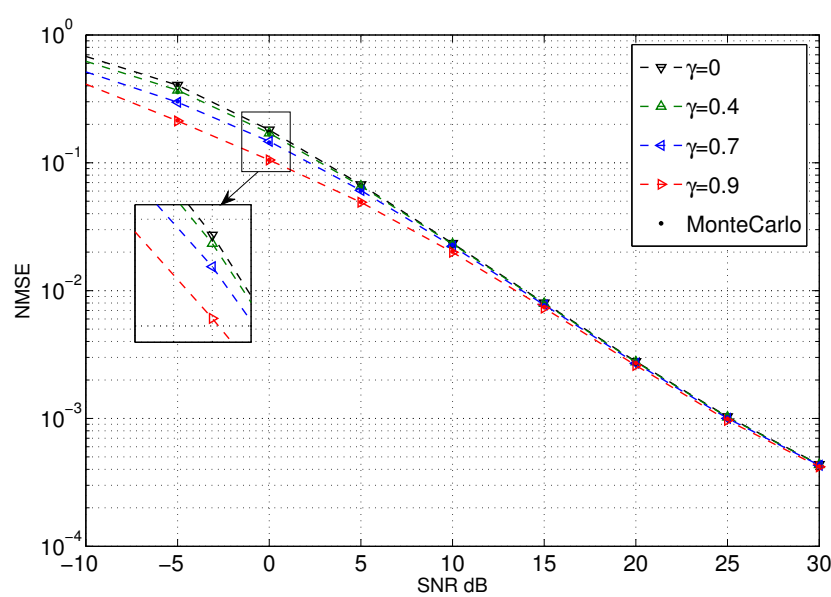

Figure 3. FBMC-QAM performance with PHYDYAS prototype filter and $\rho=0, \gamma=0.4,0.7,0.9$

To sum up, we consider both the SIMO correlation and multipaths correlation assumptions altogether. For clarity, we adopt a correlation coefficient of 0.7 and simulate the performance of FBMC-OQAM only. Fig. 4 depicts the cases of no correlation at all $(\gamma=0, \rho=0)$, only SIMO's correlation $(\gamma=0.7, \rho=0)$, only multipaths correlation $(\gamma=0, \rho=0.7)$, and the case where both SIMO and multipaths correlations exist $(\gamma=0.7, \rho=0.7)$.

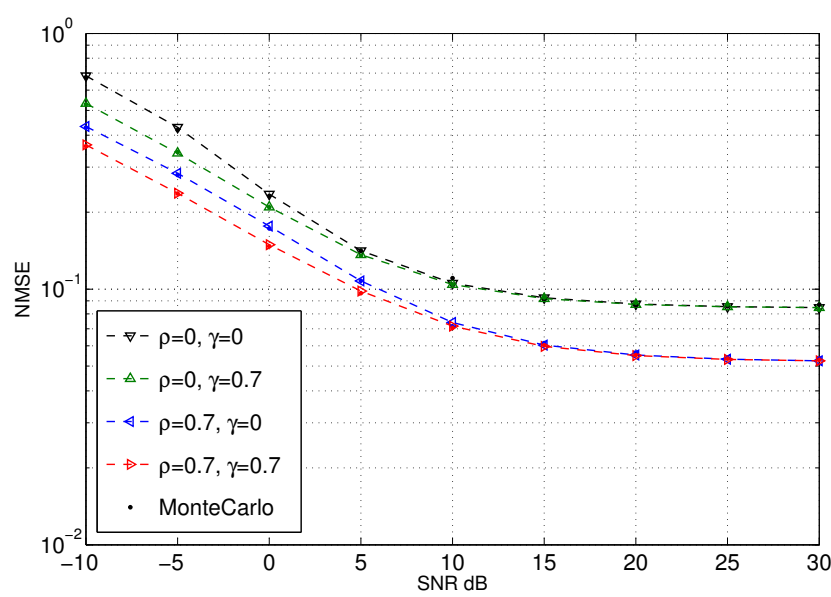

Figure 4. FBMC-OQAM performance with PHYDYAS prototype filter while adopting both a correlated multipaths and a correlated antennas assumption

\section{CONCLUSION}

In this paper, the TD-LMMSE channel estimation technique, which suits high mobility scenarios, has been analyzed for FBMC-(O)QAM based waveforms. This has been made by first proposing a generalized analytical development of the TD-LMMSE. The fact that a comb type pilot pattern was adopted means that the interference is coming only from adjacent subcarriers in the frequency domain and not from the time domain. Hence, PHYDYAS filter has shown better performance than Hermite and RRC filters since it has better frequency localization. It has been also shown that FBMCQAM offers better performance in the high speed scenarios due to the fact that the subcarriers spacing is twice that of FBMC-OQAM and OFDM, hence there is less ICI. Exploiting multipaths and multiantennas correlation have enhanced all waveforms performance. This was verified by Monte Carlo simulations. Future works include enhancing the TD-LMMSE performance for FBMC-OQAM by proposing ICI cancellation schemes.

\section{ACKNOWLEDGMENT}

This work has been performed within the SMARTIES project within the ELSAT2020 program supported by FEDER, the French State and the Haut de France Region and also the X2RAIL-1 project from H2020 SHIF2RAIL program.

\section{REFERENCES}

[1] R. Nissel et al., "Filter bank multicarrier modulation schemes for future mobile communications," IEEE Journal on Selected Areas in Communications, 2017.

[2] H. Nam et al., "A new filter-bank multicarrier system with two prototype filters for qam symbols transmission and reception," IEEE Transactions on Wireless Communications, vol. 15, no. 9, pp. 5998-6009, 2016.

[3] G. Fettweis et al., "Gfdm-generalized frequency division multiplexing," in Vehicular Technology Conference, 2009. VTC Spring 2009. IEEE 69th. IEEE, 2009, pp. 1-4.

[4] V. Vakilian et al., "Universal-filtered multi-carrier technique for wireless systems beyond lte," in Globecom Workshops (GC Wkshps), 2013 IEEE. IEEE, 2013, pp. 223-228.

[5] G. Berardinelli et al., "Generalized dft-spread-ofdm as $5 \mathrm{~g}$ waveform," IEEE Communications Magazine, vol. 54, no. 11, pp. 99-105, 2016.

[6] J.-P. Javaudin et al., "Pilot-aided channel estimation for ofdm/oqam," in Vehicular Technology Conference, 2003. VTC 2003-Spring. The 57th IEEE Semiannual, vol. 3. IEEE, 2003, pp. 1581-1585.

[7] R. Nissel et al., "On pilot-symbol aided channel estimation in fbmcoqam," in Acoustics, Speech and Signal Processing (ICASSP), 2016 IEEE International Conference on. IEEE, 2016, pp. 3681-3685.

[8] E. Kofidis et al., "Preamble-based channel estimation in ofdm/oqam systems: A review," Signal Processing, vol. 93, no. 7, pp. 2038-2054, 2013.

[9] R. Nissel et al., "On the influence of doublyselectivity in pilot-aided channel estimation for fbmc-oqam," in IEEE Vehicular Technology Conference (VTC Spring), 2017, pp. 1-5.

[10] M. Fuhrwerk et al., "Scattered pilot-based channel estimation for channel adaptive fbmc-oqam systems," IEEE Transactions on Wireless Communications, vol. 16, no. 3, pp. 1687-1702, 2017.

[11] A. Kalakech et al., "Time-domain lmmse channel estimator based on sliding window for ofdm systems in high-mobility situations," IEEE Transactions on Vehicular Technology, vol. 64, no. 12, pp. 5728-5740, 2015.

[12] Y. Cocheril et al., "On the importance of the mimo channel correlation in underground railway tunnels," Journal of Communication, vol. 4, no. 4 pp. 224-231, 2009.

[13] B. Zhang et al., "Empirical correlation property of multi-path for highspeed railways in composite propagation scenario," in Antennas and Propagation \& USNC/URSI National Radio Science Meeting, 2015 IEEE International Symposium on. IEEE, 2015, pp. 91-92.

[14] A. Paier et al., "Non-wssus vehicular channel characterization in highway and urban scenarios at $5.2 \mathrm{ghz}$ using the local scattering function," in Smart Antennas, 2008. WSA 2008. International ITG Workshop on. IEEE, 2008, pp. 9-15.

[15] A. Sahin et al., "A survey on multicarrier communications: Prototype filters, lattice structures, and implementation aspects."

[16] M. Bellanger et al., "Fbmc physical layer: a primer," PHYDYAS, January, vol. 25, no. 4, pp. 7-10, 2010.

[17] S. O. Haykin, Adaptive filter theory. Pearson Higher Ed, 2013. 